\title{
Open Versus Endovascular Revascularization of Below-Knee Arteries in Patients With End-Stage Renal Disease and Critical Limb Ischemia
}

Vascular and Endovascular Surgery

2018, Vol. 52(8) 613-620

(C) The Author(s) 2018

Article reuse guidelines:

sagepub.com/journals-permissions DOI: I0.II77/I5385744|8789036

journals.sagepub.com/home/ves

@SAGE

\author{
Alexander Meyer, MD'D, Anne Schilling', Magdalena Kott', \\ Ulrich Rother, MD', Werner Lang, $\mathbf{M D}^{\dagger}$, and Susanne Regus, MD'
}

\begin{abstract}
Background: Evaluation of below-the-knee open revascularization (OR) versus endovascular revascularization (EVT) in patients with end-stage renal disease and critical limb ischemia (CLI) was performed. Patients and Methods: Seventy-seven dialysis patients with CLI and infrapopliteal involvement from 2007 to 2017 were included. Thirty-five patients received OR and 42 patients were treated with EVT. Survival, amputation-free survival (AFS) and wound-healing were evaluated. Furthermore, both groups were analyzed for differences as to anatomic (lesion length, runoff, pedal arch classification) and clinical (VSG risk score, WIfl score) characteristics. Results: Amputation-free survival (I-year AFS: OR 54.5\% vs $47.6 \%$ in EVT, 2-year AFS OR 38.3\% vs $23.9 \% \mathrm{EVT}, P=.201$ ) did not significantly differ between OR and EVT nor did the wound healing rate (29\% OR vs $31 \% \mathrm{EVT}, P=$ .532). Overall survival was noticeably poor ( $I$-year survival: $66.7 \%$ in OR and $49 \%$ in EVT, 2 -year survival OR $47.4 \%$ vs EVT $27.7 \%$; $P=.088$ ); evaluation of peripheral runoff (Rutherford score 6.9 OR vs 7.I EVT, $P=.499$ ) and pedal arch classification as well as WIfl or VSG risk score ( 9.8 OR vs $9.6 \mathrm{EVT}, P=.673$ ) could not detect significant differences as to both the groups. Treated median lesion length was significantly increased in OR patients (OR $26 \mathrm{~cm}$ vs EVT $7 \mathrm{~cm}, P<.001$ ), whereas the incidence of major adverse cardiac events was higher in EVT patients (67\% in EVT vs 40\% OR, $P=.023)$. Conclusion: OR and EVT showed comparable outcomes as to AFS and wound healing. Poor overall survival remains the determining factor in patients with ESRD having CLI. Both groups differ in terms of anatomic features as lesion length and severity of comorbidities; considering the comparable long-term outcomes, decision-making should be based on these premises; individually applied, each method can contribute to limb salvage, although the overall survival remains limited.
\end{abstract}

\section{Keywords}

end-stage renal disease, critical limb ischemia, endovascular revascularization, surgical revascularization

\section{Introduction}

Critical limb ischemia (CLI) in patients with end-stage renal disease (ESRD) is associated with poor prognosis and limited success with regard to limb salvage and wound healing. ${ }^{1-3}$ Recent CRITISCH registry data confirmed poorer outcomes of dialysis patients after revascularization during the inhospital stay compared to patients with regular renal function, whereas long-term data are still pending. ${ }^{4}$

However, within this patient subgroup, the most efficient treatment modality especially in the complex infrapopliteal region remains unclear to this date. Endovascular revascularization (EVT) is suggested as safe and feasible option considering the morbidity in a relevant part of these patients, ${ }^{5,6}$ but open revascularization $(\mathrm{OR})$ is also reported to yield reasonable results in terms of graft patency and complication rates for selected cases. ${ }^{7}$ Even in multicenter studies assessing the treatment practice of CLI, the numbers of surgically revascularized and endovascularly treated patients with ESRD remain comparatively small for sensible analysis of differences as to outcome, amputation-free survival (AFS), and complication rates between both the groups. ${ }^{4}$ Recent literature on lower extremity revascularization among dialysis patients could not confirm significant advantages of a single therapeutic method due to the limited overall survival, but selective evaluation of belowknee vessels and anatomic criteria for decision-making is lacking. ${ }^{8}$ For this reason, we performed a retrospective single-center evaluation of dialysis patients with prospective

'Department of Vascular Surgery, University Hospital Erlangen, Erlangen, Germany

\section{Corresponding Author:}

Alexander Meyer, Department of Vascular Surgery, University Hospital Erlangen, Krankenhausstraße 12, 91054 Erlangen, Germany.

Email: alexander.meyer@uk-erlangen.de 
follow-up in a tertiary hospital with infrapopliteal manifestation of CLI for a direct comparison of both treatment modalities. Our primary aim was to analyze possible differences with influence on individual choice of treatment modality between EVT and OR patients; therefore, analysis of perioperative risk profile, anatomic characteristics and occlusion morphology, and clinical success over a 10 -year period was performed. Second, OR and EVT were compared for differences in AFS and wound healing.

\section{Patients and Methods}

The study was conducted in accordance with the principles in the Declaration of Helsinki. This study was approved by the local ethics committee, and informed consent was obtained from included patients (Ethikkommission, University of Erlangen-Nuremberg, Chairman: Prof. Maas, protocol number: 262_17B, date of approval: September 18, 2017). The study is registered (http://www.clinicaltrials.gov: NCT: 03293589).

\section{Patients and Inclusion Criteria}

Consecutive patients with ESRD undergoing revascularization of the infrapopliteal segment for CLI between 2007 and 2017 were included in the present study $(\mathrm{N}=77)$. Target arteries included the infrageniculate popliteal artery posterior tibial, anterior tibial, and/or peroneal arteries as well as the pedal vessels. Within the entire study period, a total of 2511 patients with CLI and a total of 108 patients with ESRD and CLI were treated. Critical limb ischemia patients with ESRD undergoing primary amputation and conservative treatment as well as with isolated above-knee lesions were excluded. Due to the absence of general treatment guidelines for this patient subgroup, decision-making for OR versus EVT was made from 2001 to 2010 on surgeons preference after individual consideration of patient's fitness, comorbidities, and lesion morphology as shown in diagnostic angiography; it was based on multidisciplinary vascular board review from 2011 after performance of diagnostic angiography. The patients were referred to the department of vascular surgery from their home dialysis centers or departments of nephrology. All included patients were ambulatory at least on a domestic level and presented with chronic ischemia grade Rutherford 5 or 6 . Completion angiograms were obtained both after EVT and OR intraoperatively as patency control after bypass surgery. Due to the multimorbidity of this population, no routine postoperative/postinterventional angiography controls were determined; indication for reintervention was guided by clinical analysis. Patient characteristics are shown in Table 1.

\section{Current Treatment Algorithm}

Current treatment algorithm encompasses discussion of patients in a vascular board. Dependent on individual ambulatory status, angiographic findings, lesion length, and localization as well as cardiovascular risk profile decision is made for
Table I. Patient Characteristics. ${ }^{\text {a }}$

\begin{tabular}{|c|c|c|c|c|}
\hline Patients & $\begin{array}{c}\text { Overall } \\
N=77 \\
n(\%)\end{array}$ & $\begin{array}{c}\text { Open } \\
\text { Revascularization } \\
\text { (OR) } \mathrm{N}=35 \\
\mathrm{n}(\%)\end{array}$ & $\begin{array}{c}\text { Endovascular } \\
\text { Revascularization } \\
\text { (EVT) } \mathrm{N}=42, \\
\mathrm{n}(\%)\end{array}$ & $\begin{array}{c}P \\
\text { Value }\end{array}$ \\
\hline Age (years) & & & & .781 \\
\hline$<60$ & $8(10)$ & $3(9)$ & $5(12)$ & \\
\hline $60-69$ & $21(27)$ & $10(29)$ & II (26) & \\
\hline $70-79$ & $31(40)$ & $15(43)$ & $16(38)$ & \\
\hline$>80$ & $17(22)$ & $7(20)$ & $10(24)$ & \\
\hline \multicolumn{5}{|l|}{ Gender } \\
\hline Male & $53(69)$ & $19(54)$ & $34(8 I)$ & .05 \\
\hline Female & $24(31)$ & $16(46)$ & $8(19)$ & \\
\hline \multicolumn{5}{|l|}{ Comorbidities } \\
\hline Diabetes & $60(78)$ & $27(77)$ & $33(79)$ & .880 \\
\hline CAD & $61(79)$ & 27 (77) & $34(83)$ & .682 \\
\hline $\mathrm{CHF}$ & $52(68)$ & $26(74)$ & $26(63)$ & .248 \\
\hline COPD & $16(21)$ & $6(17)$ & $10(24)$ & .473 \\
\hline Smoking & $48(62)$ & $22(63)$ & $26(62)$ & .932 \\
\hline Hypertension & $77(100)$ & $35(100)$ & $42(100)$ & \\
\hline Hyperlipidemia & $54(70)$ & $24(69)$ & 30 (7I) & .785 \\
\hline Renal disease & & & & $.7 \mid 4$ \\
\hline $\begin{array}{l}\text { Diabetic } \\
\text { nephropathy }\end{array}$ & $44(57)$ & $18(5 \mathrm{I})$ & $26(62)$ & \\
\hline $\begin{array}{l}\text { Hypertensive } \\
\text { nephropathy }\end{array}$ & $17(22)$ & $10(29)$ & $7(17)$ & \\
\hline $\begin{array}{c}\text { Glomerular } \\
\text { nephritis }\end{array}$ & $7(9)$ & $3(9)$ & $4(10)$ & \\
\hline $\begin{array}{l}\text { Interstitial } \\
\text { nephritis }\end{array}$ & $3(4)$ & $2(6)$ & I (2) & \\
\hline $\begin{array}{l}\text { Toxic } \\
\text { nephropathy }\end{array}$ & $3(4)$ & None & $3(7)$ & \\
\hline Unknown & $3(4)$ & $2(6)$ & I (2) & \\
\hline
\end{tabular}

Abbreviations: $\mathrm{CAD}$, coronary artery disease; $\mathrm{CHF}$, congestive heart failure; $\mathrm{CLI}$, critical limb ischemia; COPD, chronic obstructive pulmonary disease ${ }^{a}$ Data are presented as numbers (\%).

amputation, open, or endovascular revascularization. This pathway is shown in Figure 1.

\section{End Points}

Both groups were analyzed for differences in lesion morphology (pedal arch, lesion length, runoff) as well as wound status and perioperative risk factors. For these purposes, the cardiac risk index (VSG-CRI) was calculated for each patient as proposed by the Vascular Study Group of New England for vascular surgery patients. ${ }^{9}$ Wounds were graded according to the WIfI classification system. ${ }^{10,11}$ Primary end points for comparison of both groups were the AFS, overall survival, wound healing as well as major adverse cardiac events (MACE). Reinterventions were recorded but not counted as end points, as patency rates were outside the topics of present analysis.

\section{VSG-Cardiac Risk Index}

This index was developed as vascular-specific score for estimation of perioperative adverse cardiac events, consisting of 


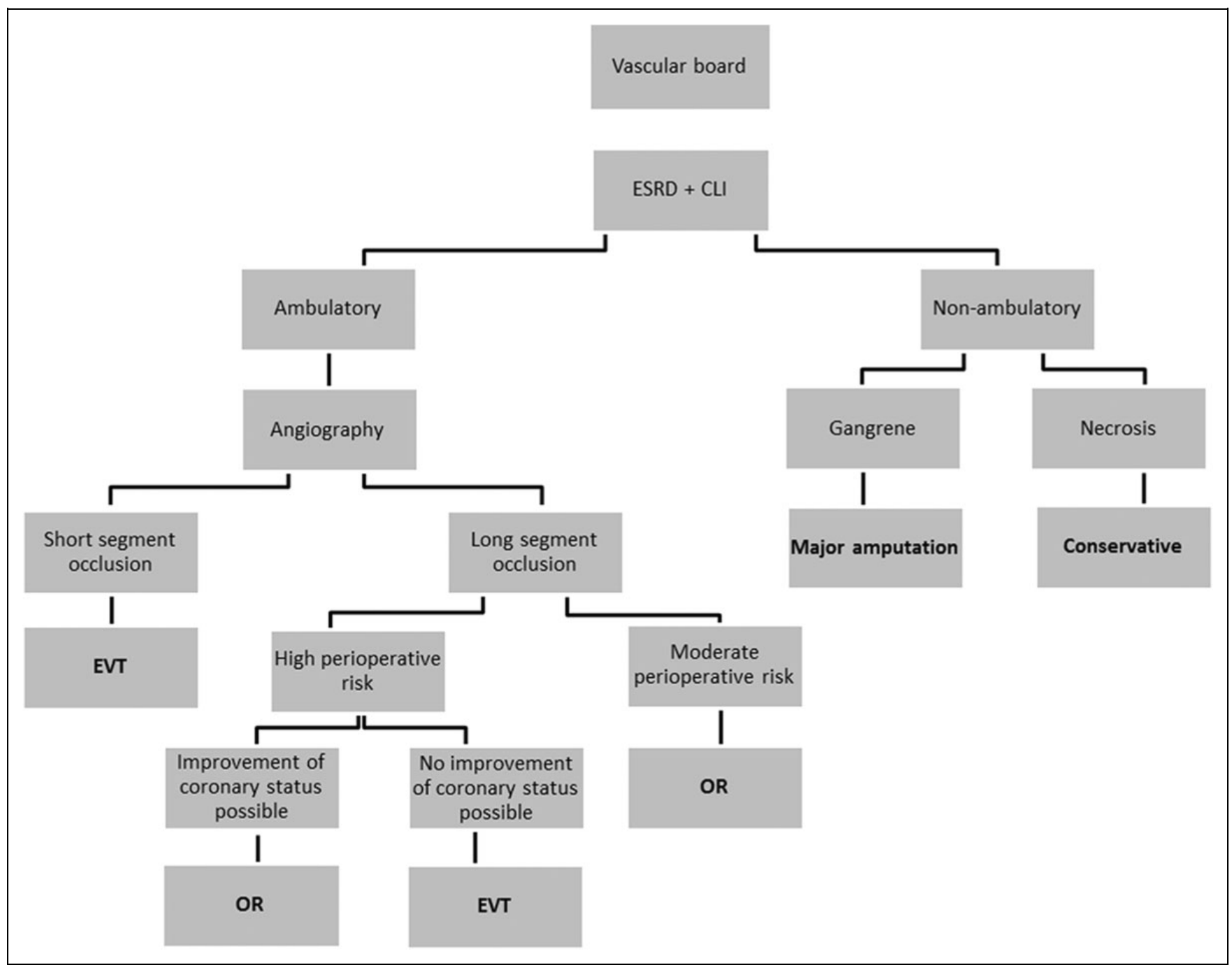

Figure I. Treatment algorithm for patients with ESRD with CLI. Short segment occlusion infrapopliteal $<10 \mathrm{~cm}$; long-segment occlusion infrapopliteal $>10 \mathrm{~cm}$; moderate perioperative risk: VSG-CRI score $\leq 6$; high perioperative risk: VSG-CRI score $>7$. CLI indicates critical limb ischemia.

several predictors (age, smoking, insulin-dependent diabetes, coronary artery disease [CAD], congestive heart failure, longterm beta-blocker therapy, chronic obstructive pulmonary disease, and creatinine $>1.8 \mathrm{mg} / \mathrm{dL}$ ), derived from regression analysis of a comprehensive study cohort. ${ }^{9}$

\section{Pedal Arch and Runoff}

Kawarada's classification of the pedal arch was used for grading of the pedal vessel patency. ${ }^{12}$ Category 1 was applied in case of fully patent pedal arch, category $2 \mathrm{a}$ was used in case of patent dorsalis pedis and occluded plantar artery, whereas category $2 \mathrm{~b}$ comprised cases with patent plantar and occluded dorsalis pedis artery. Category 3 was used in case of no patent pedal vessel. Furthermore, a modified Rutherford pedal runoff score was calculated for each patient after EVT or OR. Three arteries were evaluated for the modified runoff score: the lateral plantar artery, the medial plantar artery, and the dorsalis pedis artery. Scoring was performed according to Society of Vascular Surgery standards (0: $<20 \%$ stenosis, 1: $21 \%-49 \%$ stenosis, 2: 50\%-99\% stenosis; 2.5: <half the vessel length occluded, 3: > half the vessel length occluded). ${ }^{13}$ In case of single-vessel outflow via the peroneal artery, 1 was added to the foot score in order to reflect the indirect inflow to the pedal arch. This resulted in a summarized foot score of 0 to 10 for each patient, 10 representing the worst outflow situation via peroneal artery, and no pedal vessel patent. ${ }^{14,15}$

\section{Statistical Analysis}

Categorial data are presented as counts and percentages, continuous data as median and range in case of nonparametric distribution. Parametric data are given as mean and standard deviation (SD). Kaplan-Meier method was used to calculate AFS, survival, major amputation rate, and clinical success. Patients who died or were lost to follow-up were censored at 
the point of death or their last contact. The log-rank test was used for the comparison of Kaplan-Meier survival curves. Due to slightly skewed distribution of obtained values, nonparametric techniques were used for analyses of statistically noticeable differences within the outcome parameters. In particular, we used the Mann-Whitney $U$ test for independent samples for evaluation of interrelations between continuous variables. In case of categorial data, significance was tested by Fisher exact test and $\chi^{2}$ test. A value of $P<.05$ was considered significant. All analyses were performed using statistical software SPSS for Windows version 21 (SPSS Inc, Chicago, Illinois).

\section{Follow-Up}

Follow-up was conducted in cooperation with patient's dialysis centers and their attending physicians. Telephone consultations with dialysis physicians were determined at regular intervals. Symptom-based physical examinations and assessment of local wound situation were done and recorded for each patient's dialysis attendance, usually 3 times a week. Wound healing was defined as a WIfI-score of W0 Ix fIx and was followed by photo documentation. In the event of any deterioration, defined as downgrading of at least one level in any WIfI category, the patients were referred to the department of vascular surgery for further evaluation.

\section{Results}

\section{Basic Characteristics}

Seventy-seven patients were included. Mean age was 72.5 years (SD 7.7 years), 53 were male, and 24 were female. Median time on dialysis was 40 months (range: 1-184 months). Thirty-five patients received an open revascularization by infrapopliteal bypass, whereas 42 underwent tibial endovascular treatment. Median follow-up time was 25 months in OR patients (range: 1-119 months) and 19 months for EVT patients (range: 1-66 months). Nine (11.6\%) patients had a history of previous femoropopliteal interventions. A total of $11(14 \%)$ patients was treated from 2007 to 2010, whereas the majority (86\%) of cases was included between 2011 and 2017. Relation of OR versus EVT in the first period was $82 \%$ versus $18 \%$ in the earlier years, compared to $39 \%$ versus $61 \%$ from 2011 on.

Differences between EVT and OR group were detected with respect to gender. EVT group included significantly more men ( $54 \%$ male in OR vs $81 \%$ male EVT; $P=.05$ ). However, distribution of cardiovascular risk factors and comorbidities was equal between both groups as was the median time on dialysis (34 months OR, range 3-133 months; 43 months EVT, range 1184 months, $P=.868$ ). Patient characteristics are summarized in Table 1. Regarding the OR group, site of distal bypass anastomosis was the infragenual popliteal artery in $37 \%$, the tibiofibular trunk in $17 \%$, the peroneal artery in $14 \%$, the anterior tibial or posterior tibial artery in $11 \%$ each. Pedal vessels were chosen as recipient vessels for bypass anastomosis in $9 \%$ (Table 2). In the EVT group, the infragenual popliteal artery was used as isolated target vessel in $14 \%$, the anterior tibial artery, posterior
Table 2. Procedure Characteristics: Open Revascularization.

\begin{tabular}{lc}
\hline Bypass Surgery $(\mathrm{N}=35)$ & $\mathrm{n}(\%)$ \\
\hline Distal anastomosis & \\
Below-knee popliteal artery & $13(37)$ \\
Tibiofibular trunk & $6(17)$ \\
Anterior tibial artery & $4(\mathrm{II})$ \\
Posterior tibial artery & $4(\mathrm{II})$ \\
Peroneal artery & $5(14)$ \\
Pedal vessels & $3(9)$ \\
Proximal anastomosis & $17(49)$ \\
Common femoral artery & $7(20)$ \\
Superficial femoral artery & $1 \mathrm{I}(3 \mathrm{I})$ \\
Suprageniculate popliteal artery & $21(60)$ \\
Graft material & $14(40)$ \\
Autologous vein & \\
Prosthetic conduit &
\end{tabular}

Table 3. Procedure Characteristics: Endovascular Revascularization.

\begin{tabular}{ll}
\hline Endovascular Revascularization $(\mathrm{N}=42)$ & $\mathrm{n}(\%)$ \\
\hline Isolated target vessel & \\
Below-knee popliteal artery & $6(14)$ \\
Anterior tibial artery & $5(12)$ \\
Posterior tibial artery & $2(5)$ \\
Peroneal artery & $3(7)$ \\
Treatment of 2 below-knee vessels & $13(3 \mathrm{I})$ \\
Treatment of 3 below-knee vessels & $13(3 \mathrm{I})$ \\
Inflow EVT above knee & $13(3 \mathrm{I})$ \\
Treatment type & $42(100)$ \\
PTA &
\end{tabular}

Abbreviation: PTA, percutaneous transluminal angioplasty.

tibial, and peroneal artery in $12 \%, 5 \%$, and $7 \%$, respectively. Thirty-one percent of patients received EVT of all 3 tibial vessels. Thirty-one percent of patients underwent simultaneous in-flow angioplasty of superficial femoral artery. Procedure characteristics are depicted in Tables 2 and 3. Mean length of hospital stay was 19 days for OR (SD 12 days) and 10 days (SD 9 days) for EVT patients $(P<.01)$.

\section{Pedal Arch and Runoff}

Distribution of pedal arch categories was 1 in 14\%, 2a in 23\%, $2 \mathrm{~b}$ in $20 \%$, and 3 in $31 \%$ of patients within the OR group. EVT patients were graded as category 1 in $7 \%$, as $2 \mathrm{a}$ in $38 \%$, as $2 \mathrm{~b}$ in $7 \%$, and 3 in $45 \%$. No significant differences were found between both the groups $(P=.132)$. Likewise, evaluation of modified Rutherford runoff score revealed no differences between both groups (mean 6.9 OR SD 2.1 vs 7.1 EVT SD $1.8, P=.499$, Table 4).

\section{Lesion Length}

Lesion length was analyzed for each patient upon diagnostic preoperative resp. preinterventional diagnostic angiogram. In 
Table 4. Anatomic and Lesion Characteristics. ${ }^{a}$

\begin{tabular}{lccc}
\hline & OR & EVT & $P$ Value \\
\hline Median lesion length, cm & 26 & 7 & $<.01$ \\
Mean Rutherford runoff score & 6.9 & 7.1 & .499 \\
Pedal arch classification & & & .132 \\
I & $5(14)$ & $3(7)$ & \\
$2 \mathrm{a}$ & $8(23)$ & $16(38)$ & \\
$2 \mathrm{~b}$ & $7(20)$ & $3(7)$ & \\
3 & $1 \mathrm{I}(3 \mathrm{I})$ & $19(45)$ & \\
Unknown & $\mathrm{I}$ & $\mathrm{I}$ & \\
\hline
\end{tabular}

${ }^{\mathrm{a}}$ Data are presented as numbers (\%).

case of multiple EVTs of tibial vessels, the particularly longest lesion was counted for the evaluation. Eventual in-flow EVT of upper leg vessels was added to the tibial lesion length. OR group was associated with longer treated lesions than the EVT group. This difference was statistically significant (median 26 $\mathrm{cm}$ OR range 8-37 $\mathrm{cm}$ vs $7 \mathrm{~cm}$ EVT range $0.5-22.5 \mathrm{~cm}, P<$ .001 ; Table 4).

\section{Perioperative Risk}

All patients were retrospectively graded with the VSG-CRI for estimation of the individual perioperative risk with respect to a potential vascular reconstruction. However, mean VSG-CRI was 9.8 (SD 2.1) in OR and 9.6 in EVT (SD 2.2), rendering both groups as "high-perioperative risk" (estimated average risk of adverse cardiac outcome: $14.3 \%$ ). Therefore, differences regarding the perioperative cardiac risk could not be confirmed between both the groups. Furthermore, percentage of major adverse cardiovascular events prior to revascularization was higher in EVT group, compared to open revascularization ( $40 \%$ OR vs $67 \%$ EVT, $P=.023$ ).

\section{WIfl Score}

Application of WIfI score for classification of foot wounds yielded following results as to the estimation of 1-year major amputation risk: OR patients were attached a "high" risk of amputation in $43 \%$ of patients and a "moderate" risk in $29 \%$ of patients. Only 3 patients were attributed a "very low" or "low" risk. Endovascular patients presented with an equal relation, $43 \%$ were also classified as "high risk," $26 \%$ of patients as "moderate risk," $14 \%$ of patients as "low risk," and $10 \%$ as "very-low risk" $(P=.102)$.

\section{Long-Term Results}

Major amputation during follow-up was performed in 9 patients in the OR group and 4 patients in EVT group, resulting in a major amputation rate of $17 \%$ during follow-up for all included patients. Median time to major amputation was 11 months in OR patients (range: 2-26 months). Early amputation due to uncontrolled infection was necessary in 1 patient, whereas indication for amputation was irreversible ischemia as consequence of repeated graft occlusion in the other patients. Within the EVT subgroup, median time to major amputation was 3 months (range: 1-13 months); in these cases, indication for amputation was absence of adequate wound healing respectively failing improvement in WIfI-score after angioplasty. Major amputation rate was lower in endovascularly treated patients but not significant $(P=.073)$. Overall survival of included patients was poor. Mean survival time after treatment was only 15 months in EVT and 22 months in OR group $(P=$ .04). Comparison of Kaplan-Meier curves indicated a slightly higher, but not significant, - survival in OR group (Figure 2; $P$ $=.088)$. Analysis of the AFS showed no differences between both the groups $(P=.201)$.

Outcomes regarding wound healing after revascularization were likewise poor in both the groups. Within OR group, wound healing was observed in $29 \%$ of patients, compared to $31 \%$ in EVT patients $(P=.532)$ during follow-up. Time to wound healing was neither different between both patient subgroups $(P=.328)$.

Reintervention during follow-up was necessary in $17 \%$ of bypass patients caused by hemodynamic failure (graft occlusion or stenosis) in 5 patients and infection in 1 patient. In comparison, clinically driven redo angioplasty for restenosis during follow-up was performed in $31 \%$ of patients in EVT group. Results are summarized in Table 5.

\section{Discussion}

The combination of dialysis and CLI dramatically decreases overall survival and increases major amputation rates in surgical as well as endovascular therapeutic approach. ${ }^{2,3,16-18}$ Previous studies comparing outcomes of open and endovascular revascularization in patients with ESRD could not confirm the superiority of either therapeutic method in terms of AFS. The reduced overall survival has been suggested as main limiting factor in this demanding patient subgroup. ${ }^{8}$ Several risk factors have been derived as predictors of death and worse results of patients with ESRD. ${ }^{19}$ However decision-making and choice of individually most beneficial therapy regimen remains the crux in these patients. We therefore conducted the present retrospective study with prospective follow-up in order to analyze the outcomes in OR and EVT with special focus on anatomic features (pedal arch, runoff, and lesion length), possible differences in peritherapeutic cardiac risk and morbidity and wound healing. Such analyses including anatomic respectively angiographic characteristics as well as risk evaluation and its effects on the long-term outcome in OR and EVT have not been published so far. Nevertheless, these are suggested in the literature. ${ }^{8}$ This may give assistance in the individual choice of appropriate therapy modality in patients with ESRD presenting with CLI.

We found that distribution of relevant risk factors was similar between OR and EVT. No differences could be detected with regard to $\mathrm{CAD}$, diabetes, current smoking, chronic heart failure, and chronic pulmonary obstruction. The only remarkable finding was that MACE in patient's history was more 


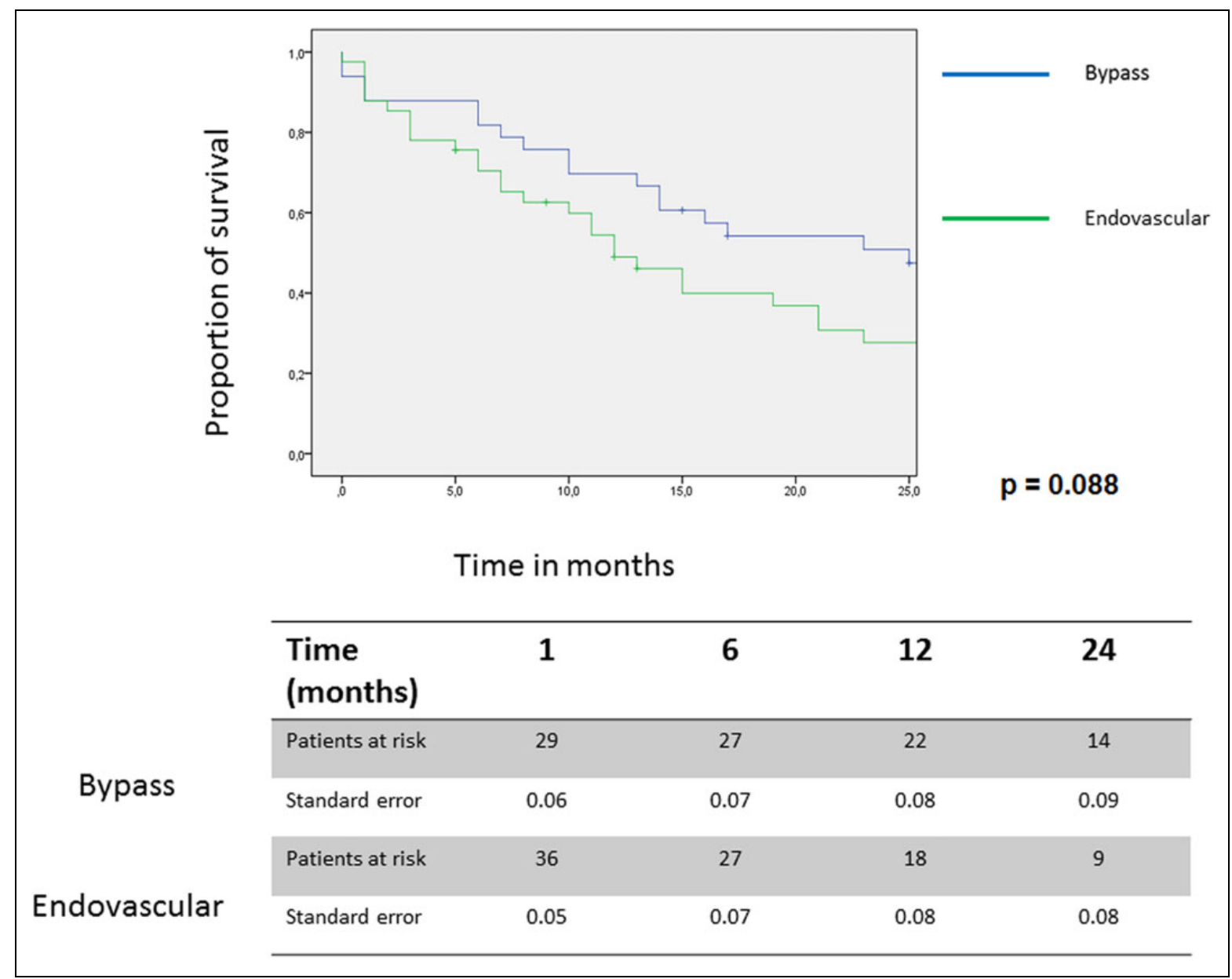

Figure 2. Comparison of Kaplan-Meier curves of dialysis patients treated with surgical and endovascular revascularization. Curves are truncated, when standard error exceeds $10 \%$.

Table 5. Summary of Results.

\begin{tabular}{|c|c|}
\hline $\begin{array}{l}\text { Amputation free survival } \\
\text { rate ( } 24 \text { months) }\end{array}$ & OR $38.3 \%$ and $23.9 \%$ EVT, $P=.201$ \\
\hline $\begin{array}{l}\text { Overall survival rate }(24 \\
\text { months) }\end{array}$ & OR $47.4 \%$ and $27.7 \%$ EVT, $P=.088$ \\
\hline $\begin{array}{l}\text { Wound healing ( } 24 \\
\text { months) }\end{array}$ & $29 \%$ OR and $31 \%$ EVT, $P=.532$ \\
\hline Pedal arch category & $\begin{array}{l}\text { No significant differences between OR } \\
\text { and EVT, } P=.132\end{array}$ \\
\hline Runoff score & Mean 6.9 OR vs 7.1 EVT, $P=.499$ \\
\hline Lesion length & $26 \mathrm{~cm}$ OR vs $7 \mathrm{~cm}$ EVT, $P<.00 \mathrm{I}$ \\
\hline WIfl score & $\begin{array}{l}\text { No differences as to I-year amputation } \\
\text { risk, }>40 \% \text { classified as "high-risk" in } \\
\text { both groups; } P=.102\end{array}$ \\
\hline VSG-CRI score & $\begin{array}{l}9.8 \text { in OR and } 9.6 \text { in EVT, } P=.673 \text {; Both } \\
\text { groups "high-risk", no significant } \\
\text { differences }\end{array}$ \\
\hline $\begin{array}{l}\text { Major adverse cardiac } \\
\text { events }\end{array}$ & $\begin{array}{l}\text { Significant more events in EVT patients } \\
(40 \% \text { OR vs } 67 \% \text { EVT, } P=.023)\end{array}$ \\
\hline
\end{tabular}

frequently observed in the EVT group. Similar distribution of comorbidities was also confirmed by calculation of VSG-CRI index. ${ }^{9}$ This index was retrospectively applied for evaluation of each patient's fitness for an OR. Hereby, both groups could equally be assessed as high risk, but significant differences were not measurable. We can therefore state that even if the presence of comorbidities were formally equal between OR and EVT group, the percentage of previous MACE was significantly elevated in the EVT group. We interpreted this as a potential sign of a more severe or advanced form of CAD and hypothesize that this may have influenced decision-making as to choice of lesser invasive EVT in cardiopulmonary "sicker" patients. Another aspect in favor of this assumption could be the observed poorer survival after treatment in EVT patients, possibly indicating a more severe impairment by comorbidities in these patients. The fact that EVT group included more men in the previous study might be due to the epidemiologically higher number of MACE in male patients ${ }^{13}$; therefore, the majority of cardiopulmonary impaired patients consisted of men who might have been rather given a less invasive EVT.

With respect to the anatomic characteristics, a remarkable finding was that neither pedal arch classification nor the Rutherford runoff score showed differences between both evaluated patient subgroups. In total, almost $40 \%$ of patients in the present study presented with fully occluded pedal arch 
compared to only $10 \%$ with fully patent pedal arch. This poor outflow to the distal foot in patients with ESRD is also reflected in the modified Rutherford runoff score (mean value OR: 6.9 vs EVT 7.1), which has been shown to correlate with the pedal arch classification in earlier studies. ${ }^{1}$ This is in congruence with recent literature, where significantly lower rates of pedal arch patency have been documented in dialysis patients. ${ }^{20,21}$ The pedal arch is generally considered an eminent factor for wound healing in CLI. ${ }^{22}$ However, the classification may be of lesser importance for decision-making in ESRD due to the high percentage of completely occluded pedal vessels in these patients. ${ }^{1}$ This fact may account for the low wound healing rates in ESRD of only $30 \%$ after revascularization. As demonstrated by the WIfI-classification, the severity and extent of wounds were comparable between bypass and endovascular group; thus, a corresponding bias regarding wound healing could be excluded. Not the outflow, but the lesion length proved to be the only relevant factor, which determined decision-making for OR or EVT in the present study. This has also been observed in infrapopliteal reconstruction in a nonESRD-specific patient cohort. ${ }^{23}$

While major amputation was more frequently (though not significant) performed in OR patients, the overall survival was considerably reduced in EVT patients. This may be attributed to the fact that patients with acute graft occlusion may be especially prone to major amputation due to irreversible and global extent of limb ischemia. Another reason might be the elevated multimorbidity of patients in EVT group, where patients possibly died for cardiac reasons prior to impending major amputation. Given the poor survival in ESRD, primary major amputation is still discussed in the literature. ${ }^{24}$ Although ESRD-specific literature on this subject is scarce, mortality rates in ESRD subsequent to major amputation may rise up to $56 \%$ at 1 year. ${ }^{25}$ While these results seem comparable to the present survival rates, ambulation and independent living after amputation is only realistic for a small proportion of patients. ${ }^{25}$ Despite low wound healing rates and reduced survival, revascularization in this patient subgroup offers at least potential preservation of walking ability and may result in better quality of life. Primary amputation may therefore be indicated in immobile patients with progressive infection.

Limitations of the present study consist in the single-center design and retrospective nature of the evaluation. However, a randomization of this patient subgroup may be challenging if not impossible in our view. The reasons might be limited overall survival, different lesion length, as well as severity of comorbidities in ESRD. In fact, life expectancy $<1$ year is often considered as an exclusion criterion in randomized trials. ${ }^{26}$ Furthermore, no routine postoperative imaging has been performed. Nevertheless, conclusions can be drawn with regard to selection of patients for surgical or endovascular revascularization. The primary aim of this study was not to evaluate the superiority of a specific therapy in patients with ESRD but to analyze OR and EVT in these patients, focusing on clinical and anatomic characteristics as well as on wound healing. Patient's clinical success after therapy was considered as relevant parameter, whereas angiographic patency was not analyzed in present multimorbid sample. Further purpose was to detect possible differences, which could influence decision-making as to the most beneficial therapeutic approach in everyday practice. In this series, we also observed a trend toward EVT therapy over the years with relation of OR versus EVT of $82 \%$ versus $18 \%$ in the earlier years compared to $39 \%$ versus $61 \%$ from 2011 on.

Against this background, we summarize that the poor patient survival remains the main determining factor in ESRD. ${ }^{8}$ The individual choice of therapeutic approach showed no significant differences with respect to AFS or wound healing; considering the observed moderate major amputation rate in our study, revascularization in this patient subgroup is still indicated and can preserve mobility and spare a considerable part of patients from major amputation. For this reason, the choice of revascularization method may be adapted to patients' risk profile, comorbidities, and lesion characteristics.

\section{Conclusion}

The present study represents a retrospective analysis of open and endovascular-treated patients with ESRD with CLI, with involvement of the lower leg vessels in everyday practice. Both groups were comparable, considering age, presence of comorbidities, peripheral runoff and risk profile at patient's admission, and wound classification. Statistical evaluation did not detect differences as to AFS or wound healing. Basis of decision-making as to choice of therapy modality turned out to be lesion characteristics and the severity of comorbidities as indicated by the higher number of MACE in EVT. EVT and OR should be considered as complementary options; individually applied, each method can contribute to limb salvage, although the overall survival remains limited.

\section{Declaration of Conflicting Interests}

The author(s) declared no potential conflicts of interest with respect to the research, authorship, and/or publication of this article.

\section{Funding}

The author(s) received no financial support for the research, authorship, and/or publication of this article.

\section{ORCID iD}

Alexander Meyer (D) http://orcid.org/0000-0002-8976-8985

\section{References}

1. Meyer A, Schinz K, Lang W, Schmid A, Regus S, Rother U. Outcomes and influence of the pedal arch in below-the-knee angioplasty in patients with end-stage renal disease and critical limb ischemia. Ann Vasc Surg. 2016;35:121-129.

2. Nakano M, Hirano K, Yamauchi Y, et al. Three-year clinical outcome after infrapopliteal angioplasty for critical limb ischemia in hemodialysis patients with minor or major tissue loss. Catheter Cardiovasc Interv. 2015;86(2):289-298. 
3. Kumada Y, Nogaki H, Ishii H, et al. Clinical outcome after infrapopliteal bypass surgery in chronic hemodialysis patients with critical limb ischemia. J Vasc Surg. 2015;61(2):400-404.

4. Meyer A, Lang W, Borowski M, Torsello G, Bisdas T; CRITISCH collaborators. In-hospital outcomes in patients with critical limb ischemia and end-stage renal disease after revascularization. J Vascul Surg. 2016;63(4):966-973.

5. Kawarada O, Yokoi Y, Higashimori A, et al. Impact of end-stage renal disease in patients with critical limb ischaemia undergoing infrapopliteal intervention. EuroIntervention. 2014;10(6): 753-760.

6. Nakano M, Hirano K, Iida O, et al. Clinical efficacy of infrapopliteal endovascular procedures for hemodialysis patients with critical limb ischemia. Ann Vasc Surg. 2015;29(6):1225-1234.

7. Hoshina K, Yamamoto K, Miyata T, Watanabe T. Outcomes of critical limb ischemia in hemodialysis patients after distal bypass surgery - poor limb prognosis with stage 4 wound, ischemia, and foot infection (WIfI). Circ J. 2016;80(11):2382-2387.

8. Fallon JM, Goodney PP, Stone DH, et al; Vascular Study Group of New England. Outcomes of lower extremity revascularization among the hemodialysis-dependent. J Vasc Surg. 2015;62(5): 1183-1191.e1.

9. Bertges DJ, Goodney PP, Zhao Y, et al; Vascular Study Group of New England. The vascular study group of New England cardiac risk index (VSG-CRI) predicts cardiac complications more accurately than the Revised Cardiac Risk Index in vascular surgery patients. J Vasc Surg. 2010;52(3):674-683, 683. e1-683.e3.

10. Mills JL Sr, Conte MS, Armstrong DG, et al. The society for vascular surgery lower extremity threatened limb classification system: risk stratification based on wound, ischemia, and foot infection (WIfI). J Vasc Surg. 2014;59(1):220-234 e1-2.

11. Ward R, Dunn J, Clavijo L, Shavelle D, Rowe V, Woo K. Outcomes of critical limb ischemia in an urban, safety net hospital population with high WIfI amputation scores. Ann Vasc Surg. 2017;38:84-89.

12. Kawarada O, Fujihara M, Higashimori A, Yokoi Y, Honda Y, Fitzgerald PJ. Predictors of adverse clinical outcomes after successful infrapopliteal intervention. Catheter Cardiovasc Interv. 2012;80(5):861-871.

13. Ladwig KH, Waller C. Gender-specific aspects of coronary heart disease [in German]. Bundesgesundheitsblatt Gesundheitsforschung Gesundheitsschutz. 2014;57(9):1083-1091.

14. Kret MR, Cheng D, Azarbal AF, et al. Utility of direct angiosome revascularization and runoff scores in predicting outcomes in patients undergoing revascularization for critical limb ischemia. J Vasc Surg. 2014;59(1):121-128.
15. Rutherford RB. Reporting standards for endovascular surgery: should existing standards be modified for newer procedures? Semin Vasc Surg. 1997;10(4):197-205.

16. Yamamoto S, Hosaka A, Okamoto H, Shigematsu K, Miyata T, Watanabe T. Efficacy of revascularization for critical limb ischemia in patients with end-stage renal disease. Eur J Vasc Endovasc Surg. 2014;48(3):316-324.

17. Bianchini Massoni C, Freyrie A, Muccini N, Gargiulo M, Faggioli G, Stella A. Endovascular treatment of the infrapopliteal arteries in hemodialysis patients with critical limb ischemia: is it justified? Ann Vasc Surg. 2014;28(1):152-158.

18. Shiraki T, Iida O, Takahara M, et al. Comparison of clinical outcomes after surgical and endovascular revascularization in hemodialysis patients with critical limb ischemia. $J$ Atheroscler Thromb. 2017;24(6):621-629.

19. Rao A, Baldwin M, Cornwall J, Marin M, Faries P, Vouyouka A. Contemporary outcomes of surgical revascularization of the lower extremity in patients on dialysis. J Vasc Surg. 2017; 66(1):167-177.

20. Honda Y, Hirano K, Yamawaki M, et al. Wound healing of critical limb ischemia with tissue loss in patients on hemodialysis. Vascular. 2017;25(3):272-282.

21. Randhawa MS, Reed GW, Grafmiller K, Gornik HL, Shishehbor $\mathrm{MH}$. Prevalence of tibial artery and pedal arch patency by angiography in patients with critical limb ischemia and noncompressible ankle brachial index. Circ Cardiovasc Inter. 2017;10(5):pii: e004605.

22. Troisi N, Turini F, Chisci E, et al. Pedal arch patency and not direct-angiosome revascularization predicts outcomes of endovascular interventions in diabetic patients with critical limb ischemia. Int Angiol. 2017;36(5):438-444.

23. Patel SD, Biasi L, Paraskevopoulos I, et al. Comparison of angioplasty and bypass surgery for critical limb ischaemia in patients with infrapopliteal peripheral artery disease. Br J Surg. 2016; 103(13):1815-1822.

24. Brosi P, Baumgartner I, Silvestro A, et al. Below-the-knee angioplasty in patients with end-stage renal disease. J Endovasc Ther. 2005;12(6):704-713.

25. Serizawa F, Sasaki S, Fujishima S, Akamatsu D, Goto H, Amada N. Mortality rates and walking ability transition after lower limb major amputation in hemodialysis patients. J Vasc Surg. 2016; 64(4):1018-1025.

26. Zeller T, Baumgartner I, Scheinert D, et al. IN.PACT Amphirion paclitaxel eluting balloon versus standard percutaneous transluminal angioplasty for infrapopliteal revascularization of critical limb ischemia: rationale and protocol for an ongoing randomized controlled trial. Trials. 2014;15:63. 